\title{
Frameless robotic stereotactic biopsies: a consecutive series of 100 cases
}

\author{
Michel Lefranc, MD, Cyrille Capel, MD, Anne-Sophie Pruvot-Occean, MD, Anthony Fichten, MD, \\ Christine Desenclos, MD, Patrick Toussaint, MD, PhD, Daniel Le Gars, MD, PhD, \\ and Johann Peltier, MD, PhD
}

Department of Neurosurgery, Centre Hospitalier Universitaire Amiens, France

\begin{abstract}
OBJECT Stereotactic biopsy procedures are an everyday part of neurosurgery. The procedure provides an accurate histological diagnosis with the least possible morbidity. Robotic stereotactic biopsy needs to be an accurate, safe, frameless, and rapid technique. This article reports the clinical results of a series of 100 frameless robotic biopsies using a Medtech ROSA device.

METHODS The authors retrospectively analyzed their first 100 frameless stereotactic biopsies performed with the robotic ROSA device: 84 biopsies were performed by frameless robotic surface registration, 7 were performed by robotic bone fiducial marker registration, and 9 were performed by scalp fiducial marker registration. Intraoperative flat-panel CT scanning was performed concomitantly in 25 cases. The operative details of the robotic biopsies, the diagnostic yield, and mortality and morbidity data observed in this series are reported.
\end{abstract}

RESULTS A histological diagnosis was established in 97 patients. No deaths or permanent morbidity related to surgery were observed. Six patients experienced transient neurological worsening. Six cases of bleeding within the lesion or along the biopsy trajectory were observed on postoperative CT scans but were associated with transient clinical symptoms in only 2 cases. Stereotactic surgery was performed with patients in the supine position in 93 cases and in the prone position in 7 cases. The use of fiducial markers was reserved for posterior fossa biopsy via a transcerebellar approach, via an occipital approach, or for pediatric biopsy.

CONCLUSIONS ROSA frameless stereotactic biopsies appear to be accurate and safe robotized frameless procedures.

http://thejns.org/doi/abs/10.3171/2014.9.JNS14107

KEY WORDS robotic; biopsy; frameless; stereotactic radiosurgery

$\mathrm{S}$ TEREOTACTIC biopsy is a routine procedure performed in all neurosurgical centers. The purpose of stereotactic biopsy is to obtain an accurate histological diagnosis with the least possible morbidity. Historically, most surgeons who were trained in free-hand biopsy (in the 1970s) converted to frame-based stereotactic biopsy (in the 1980s and 1990s) because of its safety, accuracy, precision, and reliability. ${ }^{11,19}$ Frameless techniques have now been adopted by the majority of neurosurgery practitioners, although frame-based techniques are still indicated in specific settings. ${ }^{27,31}$ To date, only a few centers routinely perform robot-assisted biopsies. ${ }^{3,8,13,16,23}$ Robot- ic stereotactic biopsy needs to allow for accurate, safe, frameless, and rapid procedures by combining the advantages of frameless and frame-based techniques. However, its place remains uncertain, as only a few clinical series of robotic biopsies - based on a limited number of patients have been published. ${ }^{3,8,16,23}$ This article reports the clinical results of our first series of 100 frameless robotic biopsies.

\section{Methods}

\section{Robotic Device}

The robotic device used in this study was the ROSA 
(Medtech), a new integrated multi-application robotic platform composed of a robotic arm with 6 degrees of freedom coupled to a planning station. The robot also has haptic abilities, ${ }^{20}$ allowing it to be used as a supervisorycontrolled device as well as a shared-control device.

\section{Patient Population}

We retrospectively evaluated the first 100 cases of consecutive frameless robotic stereotactic biopsy performed in our department between April 2011 and May 2013 (67 males and 33 females; sex ratio 2.03). The study population comprised pediatric and elderly patients (median age at presentation 59 years; range $7-86$ years).

\section{Neuroimaging}

All preoperative imaging (CT and MRI) was performed without a frame or anesthesia several days before surgery. A preoperative CT scan (continuous $0.625-\mathrm{mm}$ slice thickness; no gantry; matrix size of $512 \times 512$ pixels) was acquired on the day before surgery. Flat-panel CT (FPCT) was performed with the O-arm Surgical Imaging System (Medtronic) using standard 3D acquisition (0.8$\mathrm{mm}$ slice thickness; no gantry; matrix size of $512 \times 512$ pixels). The 3-T MRI protocol (Signa HDx, GE Healthcare) used in this series was not specific to our stereotactic biopsy protocol but was part of the protocol used by our neuroradiological team for the diagnosis of brain tumors. The MRI sequences used for targeting consisted of 3D T2weighted sequence (1-mm slice thickness; no gantry; matrix size of $512 \times 512$ pixels); Gd-enhanced T2*-weighted gradient echo perfusion-weighted sequence; ${ }^{22}$ and Gd-enhanced 3D T1-weighted gradient echo sequence (continuous 1-mm slice thickness; no gantry; matrix size of $512 \times$ 512 pixels).

Postoperative CT scanning was systematically performed the day after surgery (continuous $0.625-\mathrm{mm}$ slice thickness; no gantry; matrix size of $512 \times 512$ pixels).

When the patient was referred to our institution with CT or MRI scans suitable for robotic surgery, only those examinations or sequences required for radiological diagnosis and/or surgical targeting were performed.

\section{Stereotactic Planning}

Biopsy needle targeting and trajectory determination were performed using the robot's planning software (Rosana, Medtech). The reference images were the preoperative CT scans for frameless surface registration and intraoperative FPCT scans for skin or bone fiducial marker registration. CT scans were matched with a preoperative nonstereotactic MRI data set after importing images into the planning station. Image coregistration used a rigid and linear algorithm. Accuracy of the image coregistration was checked, was able to be corrected, and was then validated by the surgeon. A specific interface showing the 2 imaging data sets was available, and validation was able to be achieved on anatomical landmarks such as ventricles, commissures, or bone. The target and trajectory were planned the day before surgery. Targeting was based on MR images. Surgical planning determined a gyral entry point to avoid a transsulcus trajectory or stripping of the ependymal wall. Target points were situated over a region of hyperperfusion, contrast enhancement, or, in the absence of either of these two signs, over a hyperintense signal on FLAIR or T2-weighted sequences. ${ }^{22}$ Figure 1 illustrates the surgical planning performed for one patient of this series.

\section{Surgical Procedure}

All procedures were performed with patients under general anesthesia.

For frameless surface registration biopsy, the robot was fitted with the MAYFIELD headrest (Integra LifeSciences Corporation), securely positioning the head attached to the robotic device by a rigid arm to avoid any mechanical movement. Automatic robotic frameless surface registration was performed. The robot is equipped with a registration system that combines robotic movements with noninvasive touch-free laser measurement for frameless patient surface registration. Around 5000 to 8000 points of the face, dorsum, edges of the nose, forehead, and temples are automatically registered. Accuracy of the registration was confirmed by the surgeon on several landmarks such as the root of the nose, internal and external canthus, temples, midline, and free landmarks chosen by the surgeon. Draping was performed. The robotic arm was automatically positioned along the planned trajectory. The robot was used during stereotactic surgery as an instrument holder (as arcs with frames). Thus, the surgeon manually passed instruments through the reducers. We did not use the haptic abilities of the robot to insert probes or electrodes during stereotactic surgeries. All instruments were then positioned and used through an adapted reducer held by the robotic arm. A 3.2-mm hole was drilled along the trajectory. The dura was opened with electrocautery applied to a bushing-guided blunt stylet, which was insulated except at its tip. A Sedan side-cut biopsy needle (10-mm specimen window; $2.5 \mathrm{~mm}$ in diameter) was placed along the trajectory. Staged biopsies (at least 2 stages) were performed to obtain as much tissue as possible and to optimize sample collection. Each stage obtained 4 "rosette" biopsy samples by rotating the side-cut needle according to the standard negative-pressure technique in 4 quadrants. When the tumor was too small to perform staged biopsy, only "rosette" biopsies were performed by rotating the side-cut needle to systematically obtain $4-8$ samples during surgery. The entire biopsy needle was then removed and the skin was closed with one No. 2-0 suture. The surgical procedure is illustrated in Fig. 2.

For frameless biopsy with fiducial marker registration, robotized registration was performed by means of invasive bone fiducial markers or skin fiducial markers. The MAYFIELD headrest was attached to the robotic device by a rigid arm to avoid any mechanical movement. An FPCT device (O-arm Surgical Imaging System) was then installed. Three positions were registered (parked to place the O-arm away from the patient's head and to give enough space for the surgery; lateral and frontal to center the O-arm for imaging of the head) to guide automatic movements of the O-shaped gantry during surgery. The fiducials were placed on patient's head in the operative position. 3D FPCT was performed and the scan was used as 


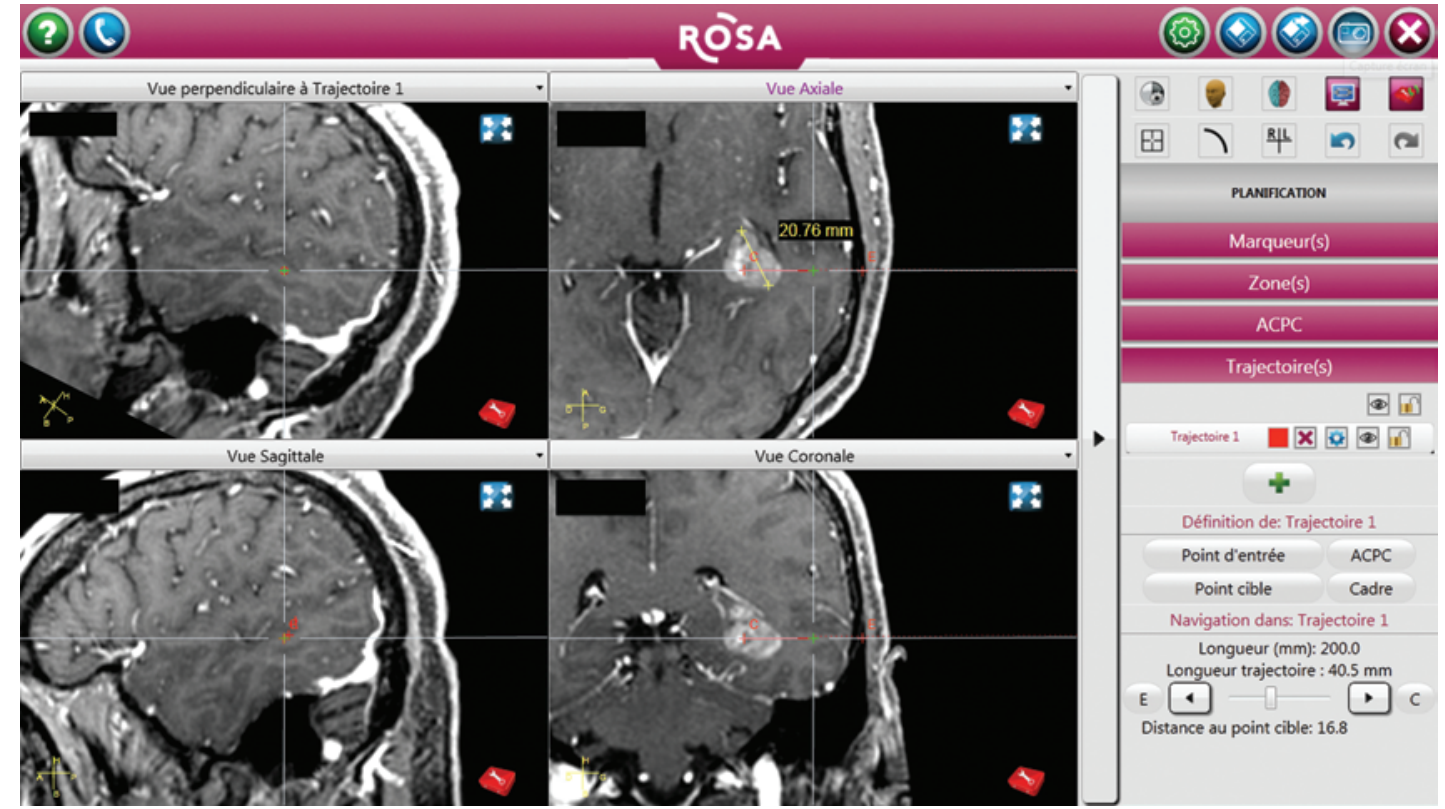

FIG. 1. Surgical planning on Rosana software. Frameless surface registration was performed in this example. The views shown (clockwise starting with the upper left image) are perpendicular, axial, sagittal, and coronal to the trajectory. Figure is available in color online only.

the reference image for registration. Automatic matching was performed with the preoperative MR images used for surgical planning. Share-controlled robotic fiducial registration was then performed. The rest of the procedure was conducted in the same way as frameless surface registration biopsy. Except for the first 5 patients, operating time was consistently less than 2 hours, which included positioning of the head, installation of FPCT and robotic devices, CT imaging data set acquisition, and draping. Fig. 3 illustrates positioning of the head when the robot was linked to FPCT.

Postoperative CT scans were matched with preoperative planning scans to check if the biopsy site was realized inside the tumor.

Patients were discharged to home on postoperative Day 1 or 2 when a satisfactory clinical status was observed. Patients returned to receive diagnostic information within 2 weeks after surgery when the definitive histology report and the multidisciplinary treatment proposal were available.

\section{Results \\ Histological Diagnosis}

A wide range of histological subtypes was observed. Details on the accuracy of tissue diagnosis are presented in Fig. 4. Neoplastic diagnoses included glioblastoma and/or Grade IV primary tumors in 51 cases, Grade I glioma in 5 cases, low-grade glioma (Grade II) in 10 cases, anaplastic glioma (Grade III) in 9 cases, brain lymphoma in 15 cases (Grade III), and metastasis in 7 cases. A nonspecific inflammatory process was observed in 2 cases, and hemorrhage and a nonspecific inflammatory process were observed in 1 case. Twenty-eight lesions were deep brain lesions; 21 lesions were situated in the corpus callosum, diencephalon, pineal region, or hypothalamus; and 8 lesions were situated in the posterior fossa, brainstem, or cerebellum. Twenty of these lesions were less than $15 \mathrm{~mm}$ in diameter.

\section{Diagnostic Yield}

After matching between preoperative planning and postoperative CT scans, all biopsy sites were inside the tumor targeted (Fig. 5). A histological diagnosis was established for 97 of the 100 biopsy targets. A nonspecific inflammatory process was described for 1 of the 3 nondiagnostic targets in a patient treated with steroids for 2 weeks that was not stopped before surgery. The final diagnosis of T-cell lymphoma was established 1 month after stopping steroid therapy.

A diagnosis of inflammatory process was also reported for a patient with a 1-cm contrast-enhancing frontobasal lesion but without hyperperfusion or tumor spectra on MRI spectroscopy. The multidisciplinary team decided not to perform a second biopsy but chose to conduct MRI every 3 months. After 9 months of follow-up, the lesion remained unchanged in terms of size and signal characteristics. The last nondiagnostic biopsy was associated with intracystic catheter placement. This 50 -year-old patient was urgently referred to our institution with a deteriorating level of consciousness and massive right hemiparesis. Preoperative MRI showed a partly cystic diencephalic lesion, the solid portion of which showed massive hemorrhagic transformation. Intracystic catheter placement allowed the patient's level of consciousness to return to normal. The histological diagnosis of the lesion was hemorrhage, necrosis, and inflammatory cells. In view of the typical appearance of the lesion and the patient's poor clinical status, the multidisciplinary team treated the tumor as glioblastoma by chemotherapy alone. The lesion rapidly progressed and the patient died within 2 months. 


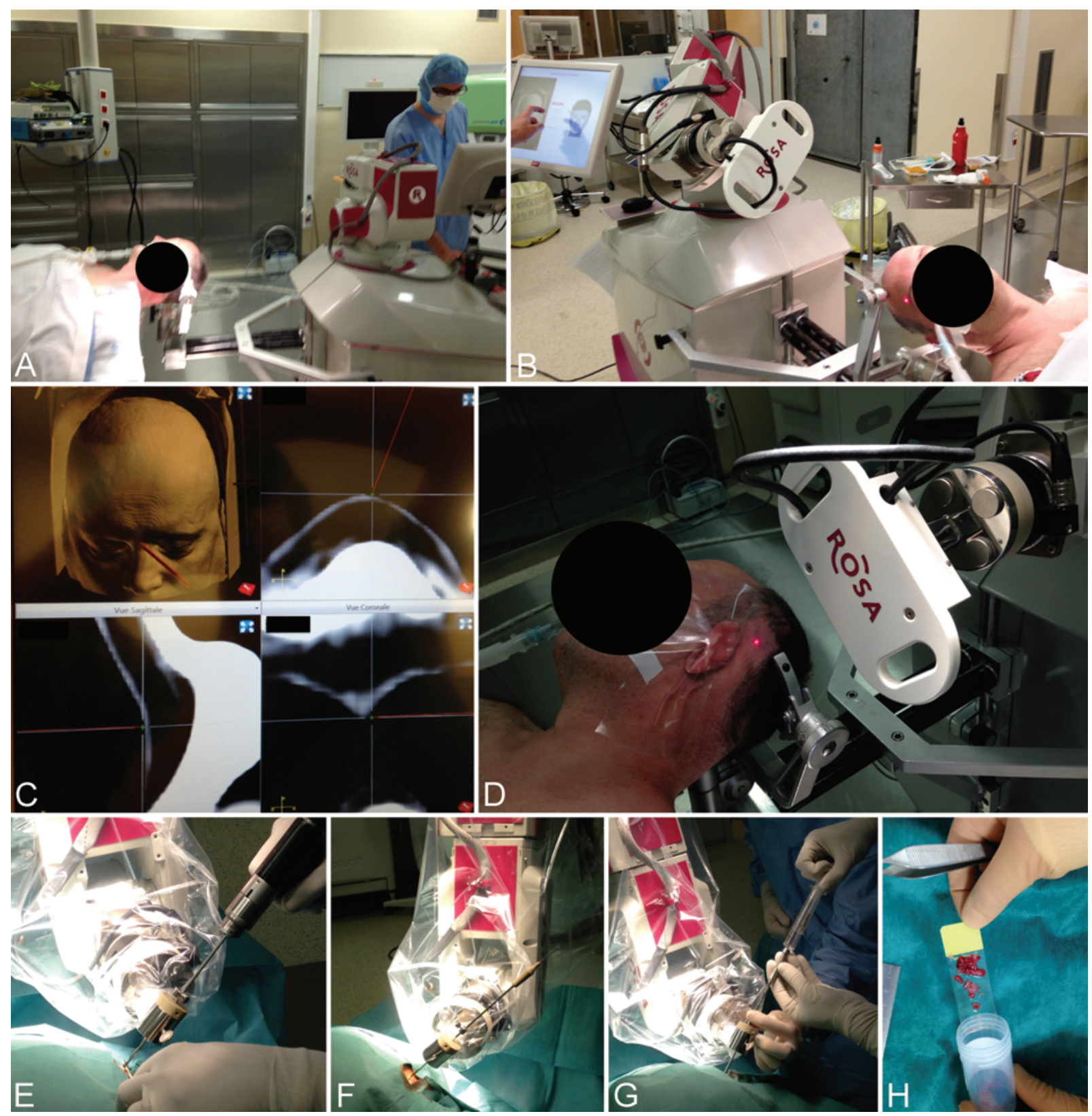

FIG. 2. Images of the ROSA robot. A: The patient is positioned for surgery. The head is fixed to the robot by means of the MAYFIELD headrest. The touch screen, on which all planning and registration data are centralized, can be seen on the right. B: The robot holds a noninvasive laser device to obtain measurements for frameless surface registration. Automatic robotic frameless surface registration is performed. The robot is equipped with a registration system that combines robotic movements with noninvasive touch-free laser measurement for frameless surface registration. Around 5000 to 8000 points of the face, dorsum, edges of the nose, forehead, and temples are automatically registered. The laser can be seen on the patient's skin. C: The accuracy of the registration is confirmed by the surgeon on several landmarks, such as roots of the nose, internal and external canthus, temples, and midline, as well as free landmarks chosen by the surgeon. D: The laser is then used to show the entry point. The hair is shaved around this point, the skin is prepared, and then draping is completed. E: A 3.2-mm hole is made. The drill follows the planned trajectory via a reducer held by the robotic arm (which is in place). The dura is opened by electrocautery applied to a bushing-guided blunt stylet that is insulated except at its tip. F: A Sedan side-cut biopsy needle (10-mm specimen window, 2.5 $\mathrm{mm}$ in diameter) is placed along the trajectory. $\mathbf{G}$ and $\mathbf{H}$ : To obtain as much tissue as possible and to optimize sample collection, staged biopsies (at least 2 stages) are performed, taking 4 "rosette" biopsies in each stage by rotating the side-cut needle with a standard negative-pressure technique in 4 quadrants. Figure is available in color online only. 

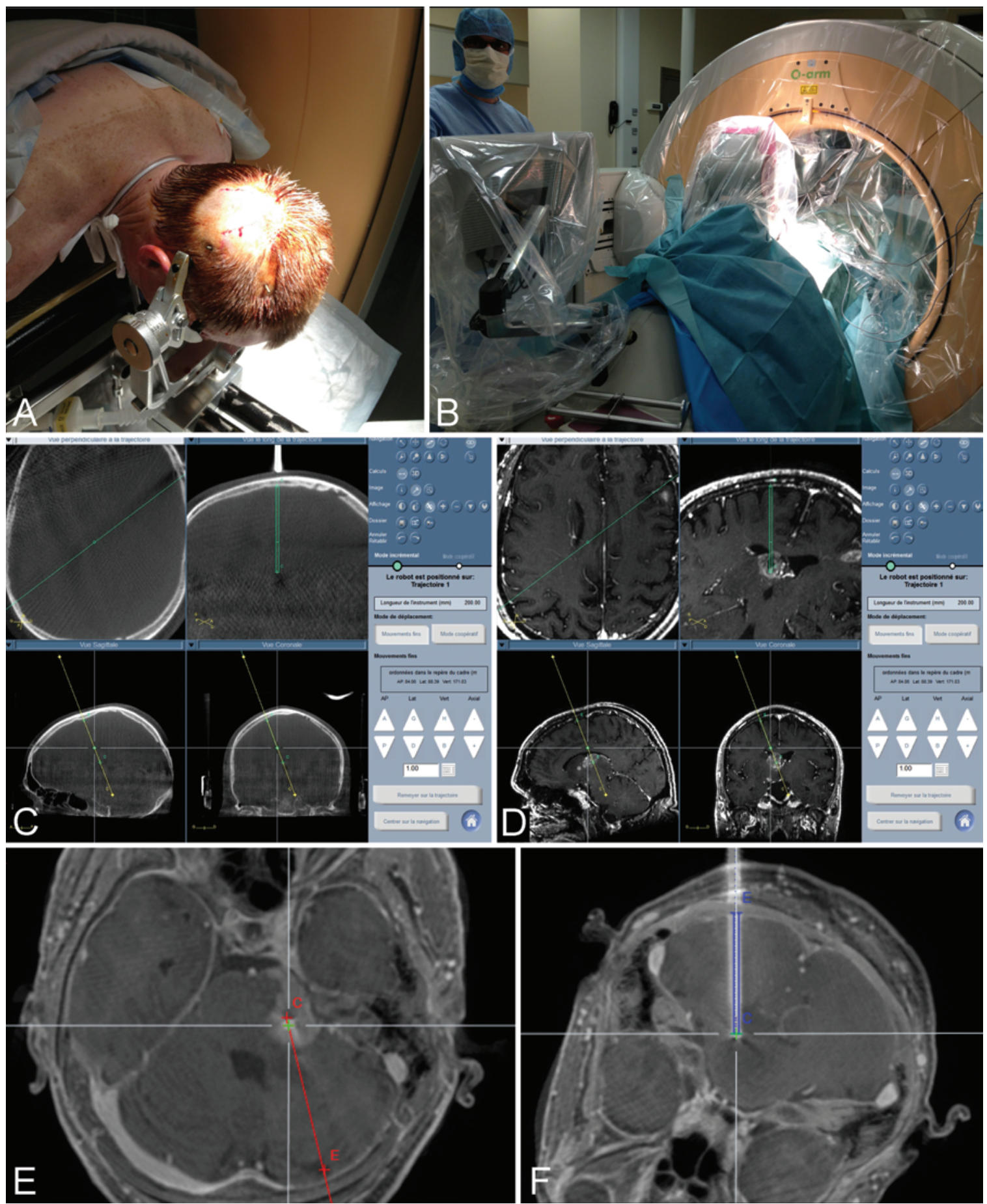

FIG. 3. Use of the robot coupled with an FPCT scanner. A: The patient is in the prone position and bone fiducials have already been placed. The head is fixed to the robot using the MAYFIELD headrest. B: Overview of the robot installation with the robotic arm and FPCT scanner in place. C and D: As a result of the matching of the intraoperative CT data with preoperative planning data on Rosana software, the accurate placement of the Sedan needle can be checked. E and F: If necessary, the correction of the robotic arm position can be performed to finally confirm the accurate position of the needle in the target. Figure is available in color online only.

\section{Bleeding Rate}

Postoperative CT demonstrated bleeding in 10 patients. Intralesional bleeding associated with transient clinical symptoms was observed in 2 cases. Minimal third ventricle hemorrhage with no clinical manifestations was ob- served after pineal tumor biopsy in 1 case. Minimal bleeding along the biopsy trajectory or at the biopsy site was observed in 7 cases. All 7 cases of bleeding were clinically asymptomatic. Fig. 6 illustrates the 2 types of bleeding observed in this series. 



FIG. 4. Upper: The various biopsy sites are shown. The whole brain was explored as well as supratentorial, deep, or infratentorial lesions. Lower: The diagnostic yield $(97 \%)$ of the robotic series and the wide range of tumor subtypes diagnosed are shown. Figure is available in color online only. V3 $=$ third ventricle. 

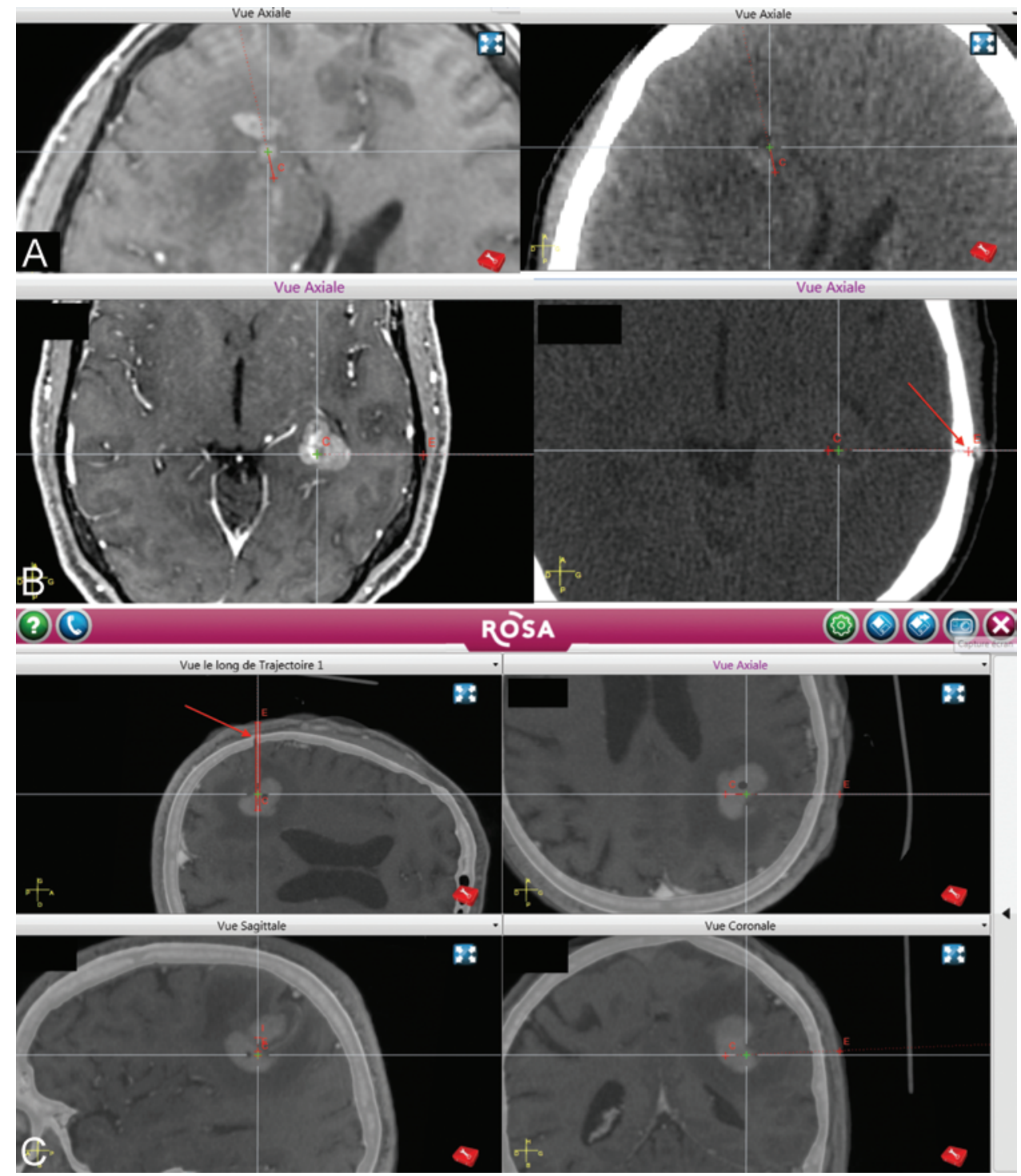

FIG. 5. Coregistration of postoperative CT data with preoperative planning data is shown. A and B: Two preoperative planning images are shown (left) and 2 postoperative CT images matched with preoperative planning are shown (right). In all cases, biopsies were obtained within the tumor targeted. C: Postoperative CT images coregistered and merged with preoperative planning images. Bone print (arrow indicating the hole made during surgery) and air bubble confirming that the biopsies were obtained inside the tumor. In all 100 cases, the biopsy site was inside the tumor targeted. Figure is available in color online only.

\section{Mortality, Transient Morbidity, and Permanent Sequelae}

No mortality, infectious morbidity, or permanent sequelae related to stereotactic biopsy were observed.

Six patients experienced transient morbidity. Transient deterioration of preexisting hemiparesis and oculomotor palsy (Parinaud syndrome) occurred in 1 patient with a pontine tumor. The patient's previous neurological status recovered within 10 days following stereotactic biopsy. The tumor was a large B-cell lymphoma. After 30 months of follow-up, the patient was still alive with no neurological deficit.

In another case, the patient was followed for a very large biparietal-pineal-brainstem and intraventricular (third ventricle and lateral ventricles) lesion discovered in the context of acute hydrocephalus (initially treated by endoscopic third ventriculostomy), which was associated with oculomotor palsy and severe tetraparesis. No clinical changes were observed immediately after stereotactic biopsy, but the day after the biopsy the patient suffered deterioration of his level of consciousness. CT scanning showed triventricular hydrocephalus due to obstruction of the third ventriculostomy by hemorrhage. An external shunt was placed, leading to recovery of a normal level of consciousness and the patient's previous clinical status. The lesion was a WHO Grade IV primitive neuroectodermal tumor. However, after several days, the patient experienced rapid clinical deterioration and died.

Transient deterioration of preexisting hemiparesis was demonstrated in 3 cases. All biopsies were performed over the motor gyrus. Transient deterioration of preexisting 

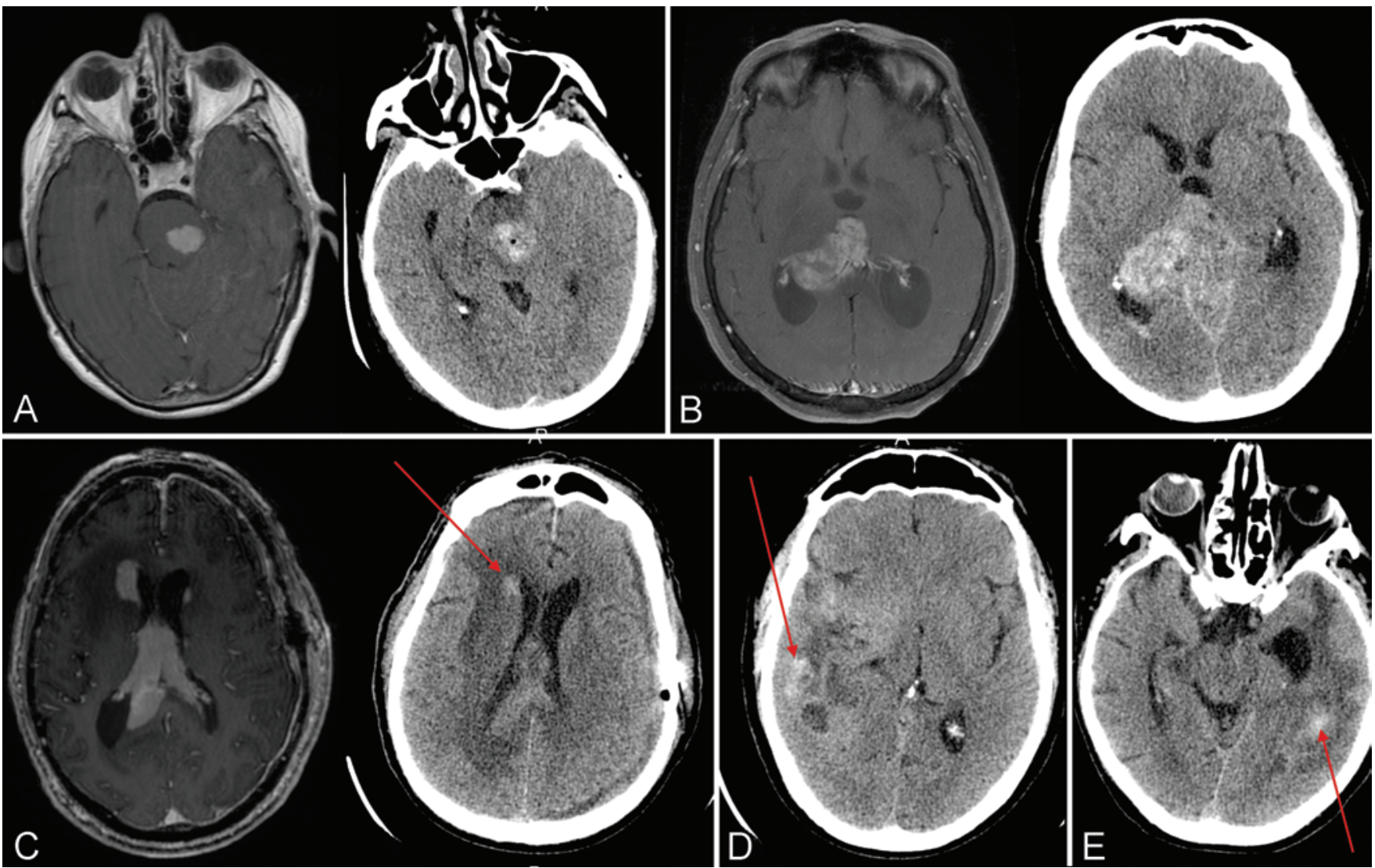

FIG. 6. Representative postoperative CT scans showing the 2 types of bleeding observed in this series. A and B: Images revealing the 2 intralesional hemorrhages that were responsible for transient clinical deterioration. C: Images illustrating the minimal bleeding (arrows) not associated with any clinical changes after biopsy. D and E: Postoperative CT scans demonstrating 2 other asymptomatic hemorrhages (arrows). Figure is available in color online only.

hemiparesis (lesion involving the postcentral gyrus) was observed in 1 case. These symptoms resolved completely within 72 hours.

Finally, only 2 cases of transient deterioration were associated with intralesional bleeding. All other clinical complications were not related to hematoma documented on postoperative CT. These symptoms were therefore probably related to brain shift and/or reflex edema following biopsy.

\section{Technical Details}

Stereotactic biopsy was performed with the patient in the supine position in 92 cases and in the prone position in 8 cases. The robot was easy to use regardless of the patient's position. However, surface registration was impossible when the patient was positioned prone because the robotic arm cannot register the face in this position. We used fiducial marker registration for these cases. The prone position was used for 2 types of biopsies: the transcerebellar approach (7 cases) and the pure occipital approach (1 case) (Table 1). Intraoperative FPCT was not necessary in the cases of fiducial marker registration. However, it facilitated the procedure by avoiding the need to place markers and create CT scan markers in place before the surgery. Imaging registration was performed with the patient in the operating position under general anes- thesia, which facilitated the biopsy procedure. FPCT reference imaging was performed with the patient in place and surgery was planned on the basis of the preoperative MRI. The images were registered on the planning software and fused with the preoperative MR images. The other main advantage of FPCT was that it allowed correction, whenever necessary, of the needle position by means of micromovement of the robot, and it also allowed intraoperative control of the needle position. Although the surgical procedure essentially took the same time, the operating room was occupied for significantly longer due to installation and scanning. Intraoperative FPCT was reserved for pediatric cases because it limits the number of preoperative imaging examinations that can be stressful for children. A transcerebellar approach was used for posterior fossa biopsy and very small deep lesions, for which intraoperative FPCT was also preferred.

The operating time for the last 50 frameless robotic biopsies was systematically less than 1 hour, including positioning of the head, installation of the robot, and draping, and less than 2 hours when the robot was coupled with the $\mathrm{O}$-arm Surgical Imaging System and bone fiducial registration.

\section{Discussion}

The ROSA robot is a new integrated multi-application 
TABLE 1. Summary of biopsy-related information

\begin{tabular}{lcc}
\hline \multicolumn{1}{c}{ Factor } & $\begin{array}{c}\text { Supratentorial } \\
\text { Biopsy }\end{array}$ & $\begin{array}{c}\text { Posterior Fossa } \\
\text { Biopsy }\end{array}$ \\
\hline Overall & 92 & 8 \\
\hline Site of biopsy & & \\
\hline Lobar & 71 & NA \\
\hline Deep* & 21 & NA \\
\hline Brainstem & NA & 6 \\
\hline Cerebellum & NA & 2 \\
\hline Robotized frameless registration & & \\
\hline$\quad$ Surface registration & 83 & 7 \\
\hline$\quad$ Fiducial markers & 9 & 1 \\
\hline Patient position & & 7 \\
\hline$\quad$ Supine & 91 & 8 \\
\hline$\quad$ Prone & 1 & \\
\hline Intraop use of FPCT $\dagger$ & 17 & \\
\hline NA $=$ not applicable. \\
* Diencephalon, corpus callosum, pineal region, or hypothalamus. \\
$\begin{array}{l}\text { Intraoperative FPCT was reserved for pediatric patients, cases of posterior } \\
\text { fossa biopsy via a transcerebellar approach, and cases of very small deep } \\
\text { lesions. }\end{array}$
\end{tabular}

platform. It is simultaneously a supervisory-controlled and a shared-control robotic device.

The aim of this study was to analyze the results of our first 100 robotic stereotactic biopsies. To our knowledge, this study represents the largest published series of robotic stereotactic biopsies. Our series shows that the diagnostic accuracy of robotic stereotactic biopsy is at least equivalent to that of conventional frame-based and frameless stereotactic procedures. ${ }^{1,2,4,6,7,14,15,18,25,27,28,31,33}$ The wide range of tumor subtypes diagnosed in the present series confirms the high rate of accurate histological diagnosis. No mortality or permanent morbidity were observed. There was no control group in our study to really compare stereotactic procedures. However, we report slightly less permanent morbidity than other big stereotactic biopsy series. ${ }^{1,2,4,6,7,14,15,18,25,27,28,31,33}$ In our opinion, our series shows that ROSA robot stereotactic biopsy allows safe stereotactic biopsy procedures.

In our opinion, the ROSA device presents several advantages. First, it is easy to use. The robot was rapidly accepted by all neurosurgeons in the department. The data reported here are based on the experience of 4 senior neurosurgeons with only 1 surgeon specialized in functional and stereotactic neurosurgery. Second, the device can be used to perform all types of registration: frame-based registration, ${ }^{21}$ frameless fiducial marker (skin or bone) registration, and frameless surface registration; this allows for the most ergonomic option for each patient with no limitation in terms of the patient's body or head position. As demonstrated in the present series, the prone position was used for the transcerebellar approach based on skin or bone fiducial marker registration. Imaging with the markers in place must be performed in these cases ${ }^{17}$ either before surgery or, as in the present series, by intraoperative CT. Third, like all-frameless stereotactic procedures, the major advantage of robotic stereotactic procedures is be- ing able to separate imaging from the actual surgical procedure. ${ }^{5,12,23}$ Surgical planning allows determination of a gyral entry point, thereby avoiding a transsulcus trajectory or the stripping of the ependymal wall, which represent the highest risks of morbidity associated with stereotactic trajectories. ${ }^{34}$ Last, the operating time is reduced to about 1 hour in the absence of intraoperative FPCT, which is similar to the operating time of a biopsy performed with a navigation system.

The main drawback of the device is its dimensions (the robot must be placed relatively close to the patient's head and occupies a space almost equivalent to that of a human being in the operative field) and, like all devices, it requires a learning curve. However, the learning curve associated with this device is short. The use of this device is similar to that of a navigation system and the surgeon only needs to learn how to use the robotic arm.

Robotic surgery has to allow for accurate, safe, frameless, and rapid procedures by combining the advantages of both frameless and frame-based procedures. Frameless biopsy is preferred because of its ergonomic advantages and because it facilitates surgical workflow. ${ }^{2,710,33}$ Frameless stereotactic biopsy is widely used for supratentorial lesions and lesions larger than $15 \mathrm{~mm} \cdot{ }^{1,2,26-28,31}$ In many neurosurgery departments, frame-based biopsy is reserved for procedures that cannot be safely performed by a frameless approach. Most neurosurgical teams still prefer framebased biopsy for brainstem lesions, small deep lesions, and lesions close to highly vascular areas, such as pineal lesions. ${ }^{27,31}$ This preference may be based on the slight but significant difference in terms of accuracy between the two techniques that, in our opinion, could explain the lower diagnostic yield of frameless biopsy for very small and posterior fossa lesions. ${ }^{2}$ Although frame-based stereotaxy is reliable, accurate, and safe even for the deepest and smallest lesions, ${ }^{8,9,23,24,29-31}$ it clearly complicates the surgical workflow. Dedicated imaging must be performed on the day of surgery with the frame in place, which is time consuming. The patient is awake and, for many neurosurgical teams, high-field MRI or CT is not available in the operating room; therefore this method may require transfer of the patient to the radiology department for scanning and return to the operating room for biopsy. ${ }^{7}$ As demonstrated by the present study, an accurate diagnosis can be obtained for lesions of all sizes in the whole brain, as diagnoses were obtained for all of the 20 lesions smaller than $15 \mathrm{~mm}$. The robotic device was used for 8 brainstem biopsies and histological diagnoses were established in every case, with only 1 case of transient clinical worsening, a result at least comparable to that obtained in frame-based biopsy series in terms of accuracy and morbidity.,24,30

Finally, the robot is easily combined with intraoperative CT, which can be used as reference imaging. With intraoperative FPCT, the needle in position can be coregistered with preoperative planning images, which allows the surgeon to confirm that the needle is accurately placed. ${ }^{21}$ Moreover, because of the robot's micromovement ability, we can correct the robotic arm position if an error is measured. Finally, intraoperative CT data coregistered to preoperative planning data can confirm the accurate position of the needle inside the target during the surgery. 
The diagnostic accuracy and the safety of the ROSA robot appear to be at least equivalent to those of the other robotic devices currently available on the market. The present series is the largest series of robotic biopsies and compares favorably with other published series. SurgiScope (Intelligent Surgical Instruments \& Systems) was used in a large series with $97.8 \%$ diagnosis rate, $2 \%$ rate of transient morbidity, and $2 \%$ rate of permanent sequelae, but no information was provided concerning the topographic sites of the tumors. ${ }^{3}$ The MKM (Carl Zeiss) was used successfully in 23 patients with a diagnostic yield of $95 \%$ and $5 \%$ rate of transient morbidity,,$^{32}$ even for very small lesions, but no information on the topographic localization of the lesions was provided. There have been no reports of large series involving the use of neuromate (Renishaw). However, there are reports of the use of this device in 17 brainstem biopsies, with a diagnostic yield of $86 \%(100 \%$ after a second procedure that was necessary for 2 patients), permanent sequelae in $6 \%$ of patients, and transient morbidity in $13 \%$, and in 17 stereotactic pineal biopsies, with a diagnostic yield of $99 \%$ and transient morbidity in $6 \% .{ }^{16,23}$ To our knowledge, no studies have been published concerning biopsies using the Pathfinder (Prosurgics Ltd.).

The ROSA device appears to have 4 main advantages over the other robotic devices: 1) it can be used to perform all kinds of registration, from surface registration to frame-based registration to marker-based registration, which means it offers a wide range of options (most other devices only provide one registration mode-skin or invasive fiducial marker registration for all devices and sometimes frame-based registration); 2) it is a mobile device (in contrast with the SurgiScope and MKM); 3) it can be easily coupled with intraoperative imaging (no modification in terms of design is necessary, compared with the neuromate, MKM, and SurgiScope, which due to their designs are not compatible with intraoperative imaging); and 4) its haptic abilities facilitate its use in nonstereotactic surgery procedures (these haptic properties allow the robot to be used as a haptic endoscope holder for ventricular endoscopy, transnasal endoscopy, and keyhole procedures). This multi-application ability may make the device more cost effective, as it is not exclusively devoted to stereotactic procedures.

\section{Conclusions}

In this large, single-center series, robotic stereotactic biopsy appeared to be a safe and effective way of establishing a histological diagnosis. In our opinion, the ROSA robot combines the advantages of frameless and framebased stereotactic surgery.

\section{References}

1. Air EL, Leach JL, Warnick RE, McPherson CM: Comparing the risks of frameless stereotactic biopsy in eloquent and noneloquent regions of the brain: a retrospective review of 284 cases. Clinical article. J Neurosurg 111:820-824, 2009

2. Barnett GH, Miller DW, Weisenberger J: Frameless stereotaxy with scalp-applied fiducial markers for brain biopsy procedures: experience in 218 cases. J Neurosurg 91:569-576, 1999
3. Bekelis K, Radwan TA, Desai A, Roberts DW: Frameless robotically targeted stereotactic brain biopsy: feasibility, diagnostic yield, and safety. Clinical article. J Neurosurg 116:1002-1006, 2012

4. Bernays RL, Kollias SS, Khan N, Brandner S, Meier S, Yonekawa Y: Histological yield, complications, and technological considerations in 114 consecutive frameless stereotactic biopsy procedures aided by open intraoperative magnetic resonance imaging. J Neurosurg 97:354-362, 2002

5. Bucholz R, McDurmont L: The history, current status, and future of the StealthStation treatment guidance system, in Lozano AM, Gildenberg PL, Tasker RR (eds): Textbook of Stereotactic and Functional Neurosurgery. Berlin: Springer, 2009, pp 543-565

6. Chen CC, Hsu PW, Wu TWE, Lee ST, Chang CN, Wei KC, et al: Stereotactic brain biopsy: single center retrospective analysis of complications. Clin Neurol Neurosurg 111:835-839, 2009

7. Dammers R, Haitsma IK, Schouten JW, Kros JM, Avezaat CJJ, Vincent AJPE: Safety and efficacy of frameless and frame-based intracranial biopsy techniques. Acta Neurochir (Wien) 150:23-29, 2008

8. Dellaretti M, Reyns N, Touzet G, Dubois F, Gusmão S, Pereira JLB, et al: Stereotactic biopsy for brainstem tumors: comparison of transcerebellar with transfrontal approach. Stereotact Funct Neurosurg 90:79-83, 2012

9. Dellaretti M, Touzet G, Reyns N, Dubois F, Gusmão S, Pereira JLB, et al: Correlation between magnetic resonance imaging findings and histological diagnosis of intrinsic brainstem lesions in adults. Neuro Oncol 14:381-385, 2012

10. Dorward NL, Paleologos TS, Alberti O, Thomas DGT: The advantages of frameless stereotactic biopsy over frame-based biopsy. Br J Neurosurg 16:110-118, 2002

11. Elder JB, Amar AP, Apuzzo MLJ: Stereotactic and imageguided biopsy, in Lozano AM, Gildenberg PL, Tasker RR (eds): Textbook of Stereotactic and Functional Neurosurgery. Berlin: Springer, 2009, pp 645-662

12. Fraser JF, Schwartz TH, Kaplitt MG: BrainLab image guided system, in Lozano AM, Gildenberg PL, Tasker RR (eds): Textbook of Stereotactic and Functional Neurosurgery. Berlin: Springer, 2009, pp 567-582

13. Glauser D, Fankhauser H, Epitaux M, Hefti JL, Jaccottet A: Neurosurgical robot Minerva: first results and current developments. J Image Guid Surg 1:266-272, 1995

14. Gralla J, Nimsky C, Buchfelder M, Fahlbusch R, Ganslandt $\mathrm{O}$ : Frameless stereotactic brain biopsy procedures using the Stealth Station: indications, accuracy and results. Zentralbl Neurochir 64:166-170, 2003

15. Grossman R, Sadetzki S, Spiegelmann R, Ram Z: Haemorrhagic complications and the incidence of asymptomatic bleeding associated with stereotactic brain biopsies. Acta Neurochir (Wien) 147:627-631, 2005

16. Haegelen C, Touzet G, Reyns N, Maurage CA, Ayachi M, Blond S: Stereotactic robot-guided biopsies of brain stem lesions: experience with 15 cases. Neurochirurgie 56:363367,2010

17. Kall BA: Image reconstruction and fusion, in Lozano AM, Gildenberg PL, Tasker RR (eds): Textbook of Stereotactic and Functional Neurosurgery. Berlin: Springer, 2009, pp 335-344

18. Kim JE, Kim DG, Paek SH, Jung HW: Stereotactic biopsy for intracranial lesions: reliability and its impact on the planning of treatment. Acta Neurochir (Wien) 145:547-555, 2003

19. Kondziolka D, Lunsford LD: The role of stereotactic biopsy in the management of gliomas. J Neurooncol 42:205-213, 1999

20. L’Orsa R, Macnab CJB, Tavakoli M: Introduction to haptics for neurosurgeons. Neurosurgery 72 (Suppl 1):139-153, 2013 
21. Lefranc M, Le Gars D: Robotic implantation of deep brain stimulation leads, assisted by intra-operative, flat-panel CT. Acta Neurochir (Wien) 154:2069-2074, 2012

22. Lefranc M, Monet P, Desenclos C, Peltier J, Fichten A, Toussaint $\mathrm{P}$, et al: Perfusion MRI as a neurosurgical tool for improved targeting in stereotactic tumor biopsies. Stereotact Funct Neurosurg 90:240-247, 2012

23. Lefranc M, Touzet G, Caron S, Maurage CA, Assaker R, Blond S: Are stereotactic sample biopsies still of value in the modern management of pineal region tumours? Lessons from a single-department, retrospective series. Acta Neurochir (Wien) 153:1111-1121, 2011

24. Levivier M: Image-guided management of brain stem lesions, in Lozano AM, Gildenberg PL, Tasker RR (eds): Textbook of Stereotactic and Functional Neurosurgery. Berlin: Springer, 2009, pp 779-788

25. McGirt MJ, Woodworth GF, Coon AL, Frazier JM, Amundson E, Garonzik I, et al: Independent predictors of morbidity after image-guided stereotactic brain biopsy: a risk assessment of 270 cases. J Neurosurg 102:897-901, 2005

26. Moriarty TM, Quinones-Hinojosa A, Larson PS, Alexander E III, Gleason PL, Schwartz RB, et al: Frameless stereotactic neurosurgery using intraoperative magnetic resonance imaging: stereotactic brain biopsy. Neurosurgery 47:1138-1146, 2000

27. Owen CM, Linskey ME: Frame-based stereotaxy in a frameless era: current capabilities, relative role, and the positiveand negative predictive values of blood through the needle. J Neurooncol 93:139-149, 2009

28. Paleologos TS, Dorward NL, Wadley JP, Thomas DG: Clinical validation of true frameless stereotactic biopsy: analysis of the first 125 consecutive cases. Neurosurgery 49:830837,2001

29. Regis J, Bouillot P, Rouby-Volot F, Figarella-Branger D, Dufour H, Peragut JC: Pineal region tumors and the role of stereotactic biopsy: review of the mortality, morbidity, and diagnostic rates in 370 cases. Neurosurgery 39:907-914, 1996

30. Roujeau T, Machado G, Garnett MR, Miquel C, Puget S,
Geoerger B, et al: Stereotactic biopsy of diffuse pontine lesions in children. J Neurosurg 107 (1 Suppl):1-4, 2007

31. Smith JS, Quiñones-Hinojosa A, Barbaro NM, McDermott MW: Frame-based stereotactic biopsy remains an important diagnostic tool with distinct advantages over frameless stereotactic biopsy. J Neurooncol 73:173-179, 2005

32. Willems PWA, Noordmans HJ, Ramos LMP, Taphoorn MJB, Berkelbach van der Sprenkel JW, Viergever MA, et al: Clinical evaluation of stereotactic brain biopsies with an MKM-mounted instrument holder. Acta Neurochir (Wien) 145:889-897, 2003

33. Woodworth GF, McGirt MJ, Samdani A, Garonzik I, Olivi A, Weingart JD: Frameless image-guided stereotactic brain biopsy procedure: diagnostic yield, surgical morbidity, and comparison with the frame-based technique. J Neurosurg 104:233-237, 2006

34. Zrinzo L, Foltynie T, Limousin P, Hariz MI: Reducing hemorrhagic complications in functional neurosurgery: a large case series and systematic literature review. Clinical article. J Neurosurg 116:84-94, 2012

\section{Author Contributions}

Conception and design: Lefranc. Acquisition of data: Lefranc, Capel, Pruvot-Occean, Fichten, Desenclos. Analysis and interpretation of data: Lefranc. Drafting the article: Lefranc. Critically revising the article: all authors. Reviewed submitted version of manuscript: all authors. Approved the final version of the manuscript on behalf of all authors: Lefranc. Statistical analysis: Lefranc. Administrative/technical/material support: Lefranc, Pruvot-Occean, Fichten, Desenclos, Toussaint, Le Gars, Peltier. Study supervision: Lefranc.

\section{Correspondence}

Michel Lefranc, Department of Neurosurgery, Place Victor Pauchet, CHU Amiens, 80054 Amiens cedex 1, France. email: lefrancm@me.com. 